\title{
IMPLEMENTACIÓN DE UN SISTEMA DE TELEDIAGNÓSTICO DE TUBERCULOSIS Y DETERMINACIÓN DE MULTIDROGORRESISTENCIA BASADA EN EL MÉTODO MODS EN TRUJILLO, PERÚ
}

\author{
Luis Rodríguez ${ }^{1,2, a}$, Alicia Alva ${ }^{3, b}, J^{2}$ orge Coronel ${ }^{3, c}$, Luz Caviedes ${ }^{3, d, \dagger}$, Alberto Mendoza-Ticona $^{4, e}$, \\ Robert Gilman ${ }^{5, f}$, Patricia Sheen ${ }^{3, d}$, Mirko Zimic ${ }^{3,9}$
}

\begin{abstract}
RESUMEN
Objetivos. Implementar un sistema para el diagnóstico remoto de tuberculosis y multidrogorresistencia (MDR) usando el método Microscopic-Observation Drug Susceptibility Assay (MODS) en el Laboratorio de Micobacterias del Centro de Excelencia en Tuberculosis de Trujillo (CENEX-Trujillo). El sistema incluyó una variante de un algoritmo de reconocimiento de Mycobacterium tuberculosis recientemente reportado a partir de imágenes digitales de cultivos MODS de muestras de esputo. Materiales y métodos. Se optimizó un algoritmo de reconocimiento por medio de un reentrenamiento del modelo estadístico basado en imágenes digitales de cultivos MODS provenientes del Laboratorio de Micobacterias del CENEX-Trujillo. Se obtuvieron imágenes de 50 cultivos MODS positivos de pacientes con sospecha de tuberculosis multidrogorresistente entre enero y octubre de 2012 en el CENEX-Trujillo. Resultados. La sensibilidad y la especificidad en objetos, para reconocer cordones de tuberculosis fueron de $92,04 \%$ y de $94,93 \%$ respectivamente. La sensibilidad y la especificidad en foto, para determinar un campo positivo a tuberculoisis fueron $95,4 \%$ y de $98,07 \%$ respectivamente. Conclusiones. Los resultados demostraron la factibilidad de la implementación de telediagnóstico en lugares remotos, el cual puede contribuir con la detección temprana de tuberculosis multidrogorresistente mediante el método MODS.
\end{abstract}

Palabras clave: Insuficiencia multiorgánica; Mycobacterium tuberculosis; Tuberculosis resistente a múltiples medicamentos (fuente: DECS/BIREME).

\section{IMPLEMENTATION OF A TELEDIAGNOSTIC SYSTEM FOR TUBERCULOSIS AND DETERMINATION OF MULTI-DRUG RESISTANCE BASED IN THE MODS METHOD IN TRUJILLO, PERU}

\begin{abstract}
Objectives. To implement a system for remote diagnosis of tuberculosis and multidrug resistance (MDR) using the Microscopic-Observation Drug Susceptibility Assay (MODS) method in the Mycobacteria Laboratory, Trujillo Center of Excellence in Tuberculosis (CENEX-Trujillo). The system included a variant of an algorithm for recognition of Mycobacterium tuberculosis recently reported from digital images of MODS cultures of sputum samples. Materials and methods. The recognition algorithm was optimized using a retraining statistical model based on digital images of MODS cultures from CENEX-Trujillo. Images of 50 positive MODS cultures of patients with suspected multidrug-resistant tuberculosis were obtained between January and October 2012 in the CENEX-Trujillo. Results. The sensitivity and specificity to recognize strings of tuberculosis were $92.04 \%$ and $94.93 \%$ respectively using objects. The sensitivity and specificity to determine a positive tuberculosis field were $95.4 \%$ and $98.07 \%$ respectively using pictures. Conclusions. The results demonstrated the feasibility of the implementation of telediagnostics in remote locations, which may contribute to the early detection of multidrug-resistant tuberculosis by MODS method.
\end{abstract}

Key words: Multiple organ failure; Mycobacterium tuberculosis; Tuberculosis, multidrug-resistant (source: MeSH/NLM).

\footnotetext{
Centro de Excelencia en Tuberculosis "Luz Caviedes Rojas", Hospital Regional Docente de Trujillo. La Libertad, Perú.

Facultad de Medicina Universidad Nacional de Trujillo. La Libertad, Perú

Laboratorio de Investigación en Enfermedades Infecciosas, Laboratorio de Investigación y Desarrollo. Facultad de Ciencias y Filosofía. Universidad Peruana Cayetano Heredia. Lima, Perú

4 Instituto Nacional de Salud. Lima, Perú

ONG A.B. PRISMA. Lima, Perú.

Médico internista, neumólogo; ${ }^{\mathrm{b}}$ Bachiller en Matemática Pura; ${ }^{\mathrm{c}}$ tecnólogo médico; ${ }^{\mathrm{d}}$ bióloga; ${ }^{\mathrm{e}}$ médico infectólogo; ${ }^{\mathrm{f}}$ médico; ${ }^{\mathrm{g}}$ físico

Fallecida

Recibido: : 31-03-14 Aprobado: 06-08-14
}

Citar como: Rodríguez L, Alva A, Coronel J, Caviedes L, Mendoza-Ticona A, Gilman R, et al. Implementación de un sistema de telediagnóstico de tuberculosis y determinación de multidrogorresistencia basada en el método MODS en Trujillo, Perú. Rev Peru Med Exp Salud Publica. 2014;31(3):445-53. 


\section{INTRODUCCIÓN}

La tuberculosis (TB) es uno de los problemas de salud pública más serios en el Perú y en el mundo. Un tratamiento adecuado de la enfermedad requiere un diagnóstico temprano, además del conocimiento de la susceptibilidad a las drogas. Los esquemas actuales de tratamiento pueden verse comprometidos si no se cuenta con una prueba de susceptibilidad rápida. El Perú, como otros países, no ha podido detener el ingreso de una nueva amenaza como lo es la tuberculosis multidrogorresistente (TBMDR) ${ }^{(1)}$.

La falta de un diagnóstico temprano y la incapacidad de determinar la susceptibilidad a drogas, hacen que los tratamientos antituberculosos no sean oportunos y apropiados, lo que compromete seriamente los esfuerzos realizados por controlar esta enfermedad. Los cultivos en medios sólidos y la prueba de sensibilidad convencional (método por proporciones) siguen utilizándose para el diagnóstico, sin embargo, esto puede tomar varios meses antes de tener el informe del resultado, lo cual puede asociarse con un alto costo. Por otra parte, el método automatizado BACTEC es rápido, pero su implementación es costosa ${ }^{(2)}$.

El método MODS (Microscopic Observation Drug Susceptibility Assay) desarrollado en el Perú permite el crecimiento y reconocimiento de patrones morfológicos de la micobacteria en medio líquido y de forma rápida, directamente de una muestra de esputo, es una opción costo-efectiva, ya que en apenas 7 a 21 días se puede detectar TB y TB-MDR, lo cual constituye una prueba alternativa rápida y de bajo costo ${ }^{(3-9)}$. Este método ha sido recientemente incorporado en la lista de pruebas rápidas autorizadas por la Estrategia Nacional de Prevención y Control de Tuberculosis. Adicionalmente, es usado en laboratorios de investigación de China, India, Indonesia, Malasia, Tailandia, Vietnam, Sudáfrica, Uganda, Zimbabue, Etiopía, Malaui, Ecuador, Bolivia, Venezuela y Brasil, donde su rendimiento ha sido estudiado con resultados positivos ${ }^{(10-13)}$.

Pese a sus bondades, la prueba MODS presenta dos limitaciones importantes: (1) este método requiere el reconocimiento de patrones morfológicos característicos mediante la observación a través de un microscopio invertido, el cual es un equipo relativamente costoso, y (2) se requiere la participación de personal técnico experimentado para interpretar las imágenes microscópicas y poder reconocer los patrones característico de tuberculosis con una alta sensibilidad y especificidad.
El avance en las tecnologías de información y comunicaciones (TIC) y sus aplicaciones en salud, ha permitido el desarrollo de la llamada telemedicina. La incorporación de las TIC en los nuevos paradigmas del cuidado de los pacientes, brinda nuevas oportunidades en el diagnóstico y tratamiento oportuno, mejorando enormemente la calidad de vida de las personas con escasos recursos, o que se encuentren en zonas de difícil acceso. La conjunción de diferentes factores como el abaratamiento de los sistemas informáticos, la extensión de las redes de comunicación y el acceso a internet posibilita que el uso de las TIC en salud ofrezca posibilidades de atención sanitaria a las poblaciones más remotas ${ }^{(14,15)}$. Existen diversas experiencias previas en las que se han utilizado las TIC en la implementación de sistemas de diagnóstico remoto, creando nuevas áreas del telediagnóstico como la telecardiología, teledermatología, teleoftalmología, telepatología, telerradiología, entre otros ${ }^{(16,17)}$.

El presente estudio demuestra el uso de las tecnologías de información y comunicaciones, en particular internet, en el telediagnóstico de tuberculosis y MDR, mediante el reconocimiento remoto de las características morfológicas específicas de cultivos MODS de muestras de esputo, empleando un algoritmo ad hoc de reconocimiento de patrones, y un sistema WEB de telediagnóstico.

\section{MATERIALES Y MÉTODOS}

\section{LUGAR Y POBLACIÓN DE ESTUDIO}

El presente estudio se llevó a cabo entre los meses de enero y octubre de 2012, en el Laboratorio de Bioinformática de los Laboratorios de Investigación y Desarrollo de la Universidad Peruana Cayetano Heredia (LID-UPCH) en Lima, donde se instaló el sistema de telediagnóstico, y CENEX-TB del Hospital Regional Docente de Trujillo (HRDT). En el CENEXTB del HRDT se realizaron las pruebas MODS y la obtención de las imágenes digitales respectivas en 50 pacientes con sospecha de tuberculosis, que asistieron de manera regular al programa de tuberculosis. A las muestras procesadas con la técnica MODS también se les realizó pruebas de sensibilidad adicionales: Griess en el Laboratorio Referencial de La Libertad, Genotype MTBDRplus y prueba de sensibilidad convencional agar placa proporciones (APP) en el Instituto Nacional de Salud. El protocolo de estudio fue aprobado por los comités de ética del Hospital Regional Docente de Trujillo y de la Universidad Peruana Cayetano Heredia. 


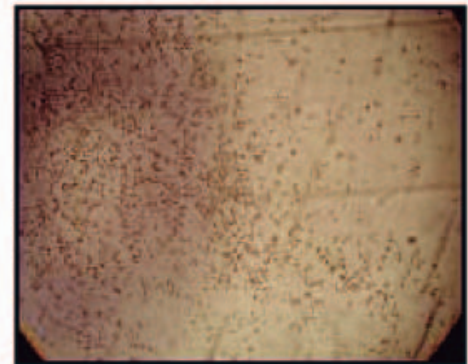

(a)

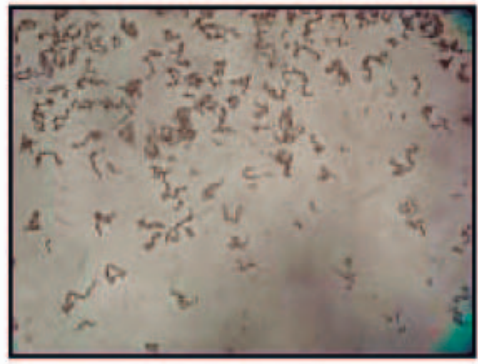

(b)

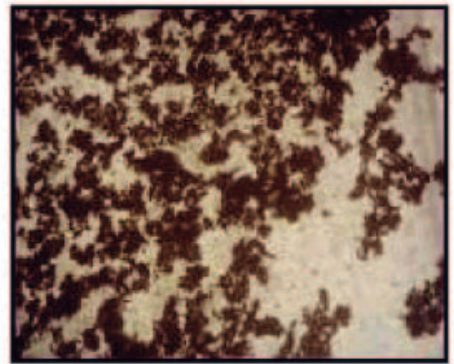

(c)

Crecimiento de Colonias de bacterias: (a) Comas (5-7 días) (b) Cordones (8-12 días) (c) Conglomerados (12- + días)

Figura 1. Etapas de crecimiento bacteriano de M. tuberculosis

\section{SISTEMA DE MAGNIFICACIÓN Y DIGITALIZACIÓN DE IMÁGENES}

Para la magnificación de imágenes se utilizó el microscopio invertido Primo Vert, (Carl Zeiss). Para la digitalización de imágenes se utilizó la cámara MiniVid de $3 \mathrm{Mp}$ con conectividad USB. La cámara de digitalización, que cuenta con un lente ocular de 10X de magnificación, se acopló directamente al receptor del ocular. Las imágenes digitalizadas se recuperaron en una computadora portátil. Las imágenes digitales fueron transmitidas a través de internet hacia el servicio WEB de telediagnóstico del LID-UPCH.

\section{ENTRENAMIENTO DEL PERSONAL TÉCNICO EN EL MÉTODO MODS Y EN EL SISTEMA DE TELEDIAGNÓSTICO}

El entrenamiento microbiológico se realizó inicialmente por dos semanas en el laboratorio de micobacterias del LID-UPCH y luego en el Laboratorio de Micobacterias del CENEX TB-HRDT. El entrenamiento incluyó aspectos microbiológicos de la prueba MODS, la digitalización de imágenes microscópicas y el manejo del sistema de telediagnóstico. Los ensayos microbiológicos se realizaron de acuerdo con las guía de usuario MODS existente en la página web (http://www.modsperu.org/) la cual contiene toda la información relevante al método MODS. En particular, se enfatizó en la observación y diferenciación de los patrones morfológicos cambiantes durante el cultivo MODS: estructuras tipo "comas" observadas entre los días 5-7; estructuras tipo "cordones" observadas entre los días 8-12, y las estructuras tipo "conglomerados" observados entre los días 12-21 (Figura 1). Es importante resaltar que las "comas" y los "conglomerados" no corresponden a patrones específicos de M. tuberculosis en un cultivo MODS.

\section{REENTRENAMIENTO DE UN ALGORITMO DE RECONOCIMIENTO DE PATRONES PARA IDENTIFICAR OBJETOS TIPO CORDONES EN UNA FOTOGRAFÍA DIGITAL DE UN CULTIVO MODS}

El algoritmo de reconocimiento de patrones recientemente publicado, fue reentrenado para optimizar su capacidad diagnóstica ante las condiciones específicas del proceso microbiológico y de digitalización de las fotografías de cultivos MODS obtenidas por el laboratorio CENEX Trujillo. El proceso general del reentrenamiento incluyó tres pasos:

Tratamiento de imagen: para mejorar el contraste de la fotografía y obtener una máxima definición en la forma de los objetos tipo "cordones" y eliminar el ruido compuesto por manchas, sombras, restos celulares y sedimentos de la muestra de esputo, entre las estructuras contaminantes más importantes. El tratamiento de la imagen consiste en dos secciones principales, el tratamiento de la imagen para resaltar los objetos de interés en la imagen de color y el conjunto de pasos que nos permiten filtrar y afinar los objetos digitales a fin de segmentar los objetos. Los cambios principales en el algoritmo previamente desarrollado incluyen ${ }^{(16)}$ :

Desenfoque gaussiano: un desenfoque gaussiano (también conocido como gaussiana suavizado) es el resultado de una imagen borrosa de una función de Gauss. Es un efecto muy utilizado en el software de gráficos, por lo general para reducir el ruido de la imagen y reducir el detalle, así se evita el ruido propio de un fondo borroso, o imágenes mal enfocadas ${ }^{(18)}$.

Binarización adaptativa: para filtrar, en la imagen recibida, el fondo de los objetos por analizar, se binariza la imagen. El método utilizado en la binarización es el algoritmo de Niblack, que adapta el valor de la umbralización en cada pixel a las 


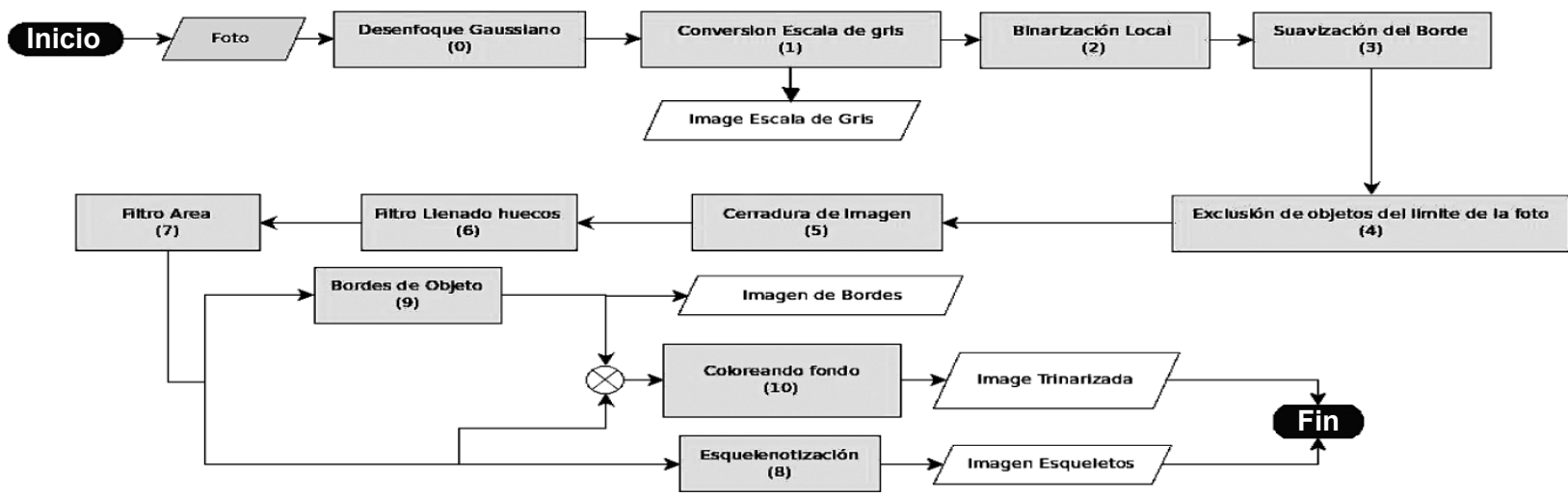

Figura 2. Diagrama de flujo del procesamiento de imagen: muestra el orden de cada proceso en el tratamiento de imagen. Los procesos (0) desenfoque gaussiano y (2) binarización local son los referentes exclusivamente a este estudio.

características locales de la imagen. Contrariamente a lo usado en diversos algoritmos con binarización global se optó por el método de Niblack, el cual corrige los artefactos ocasionados por un sistema de iluminación no uniforme en el microscopio ${ }^{(19)}$.

La Figura 2 muestra el diagrama de flujo del tratamiento de imagen e incluye los procedimientos mencionados.

Las salidas del proceso de tratamiento de imagen incluyen: la imagen en gris, el borde de los objetos,

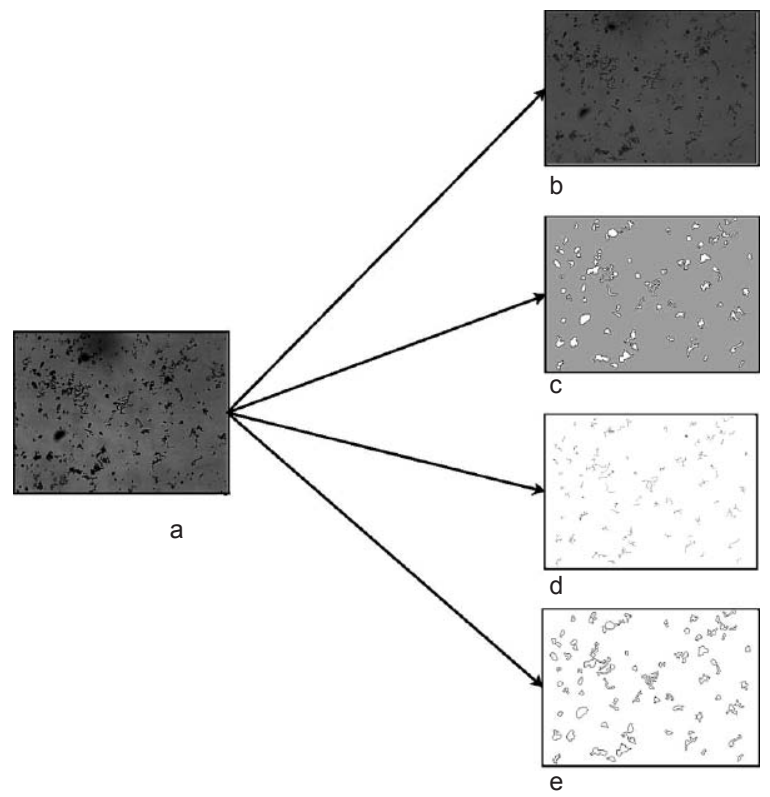

Figura 3. (0) Desenfoque gaussiano (1) conversión a Escala de gris. Procesamiento de imagen: (a) imagen original formato RGB - Input del software, las imágenes de salida del software son (b) imagen en escala de gris (c) imagen trinarizada (d) imagen de esqueletos de los objetos (e) imagen de bordes la imagen trinarizada $\mathrm{y}$, por último, la imagen del proceso de esquelenotización.

El proceso de tratamiento mencionado genera múltiples imágenes que terminan resaltando los objetos, sus bordes y los esqueletos (Figura 3 ).

\section{EXTRACCIÓN DE CARACTERÍSTICAS}

El proceso de extracción de características genera una secuencia de objetos a partir de los cuales se estiman distintos parámetros requeridos para el reconocimiento de patrones de los cordones de tuberculosis (Figura 4). Este proceso se mantuvo igual a la publicación de referencia (16). Se extrajeron 53 características de cada objeto analizado en cada fotografía de muestra de cultivo MODS. Dentro de las 53 características se incluyeron aquellas que los expertos humanos toman en consideración para clasificar un cordón de M. Tuberculosis, descritas en la Tabla 1.

Tabla 1. Extracción de características de los objetos analizados

\begin{tabular}{lc}
\hline \multicolumn{1}{c}{ Característica numérica } & $\begin{array}{c}\text { Característica } \\
\text { biológica por los } \\
\text { expertos }\end{array}$ \\
\hline Longitud & $\begin{array}{c}\text { Longitud del cordón } \\
\text { Uniformidad del } \\
\text { espesor del cordón }\end{array}$ \\
\hline $\begin{array}{l}\text { Espesor desviación estándar } \\
\text { Desviación estándar de los valores } \\
\text { de iluminación media del objeto }\end{array}$ & Refracción \\
\hline $\begin{array}{l}\text { Media de los valores de iluminación } \\
\text { del objeto }\end{array}$ & Refracción \\
$\begin{array}{l}\text { Cantidad de curvas del objeto por la } \\
\text { longitud de la mayor de las curvas }\end{array}$ & Forma \\
\hline \begin{tabular}{l} 
Perímetro / Longitud \\
\hline
\end{tabular} & Forma \\
\hline
\end{tabular}




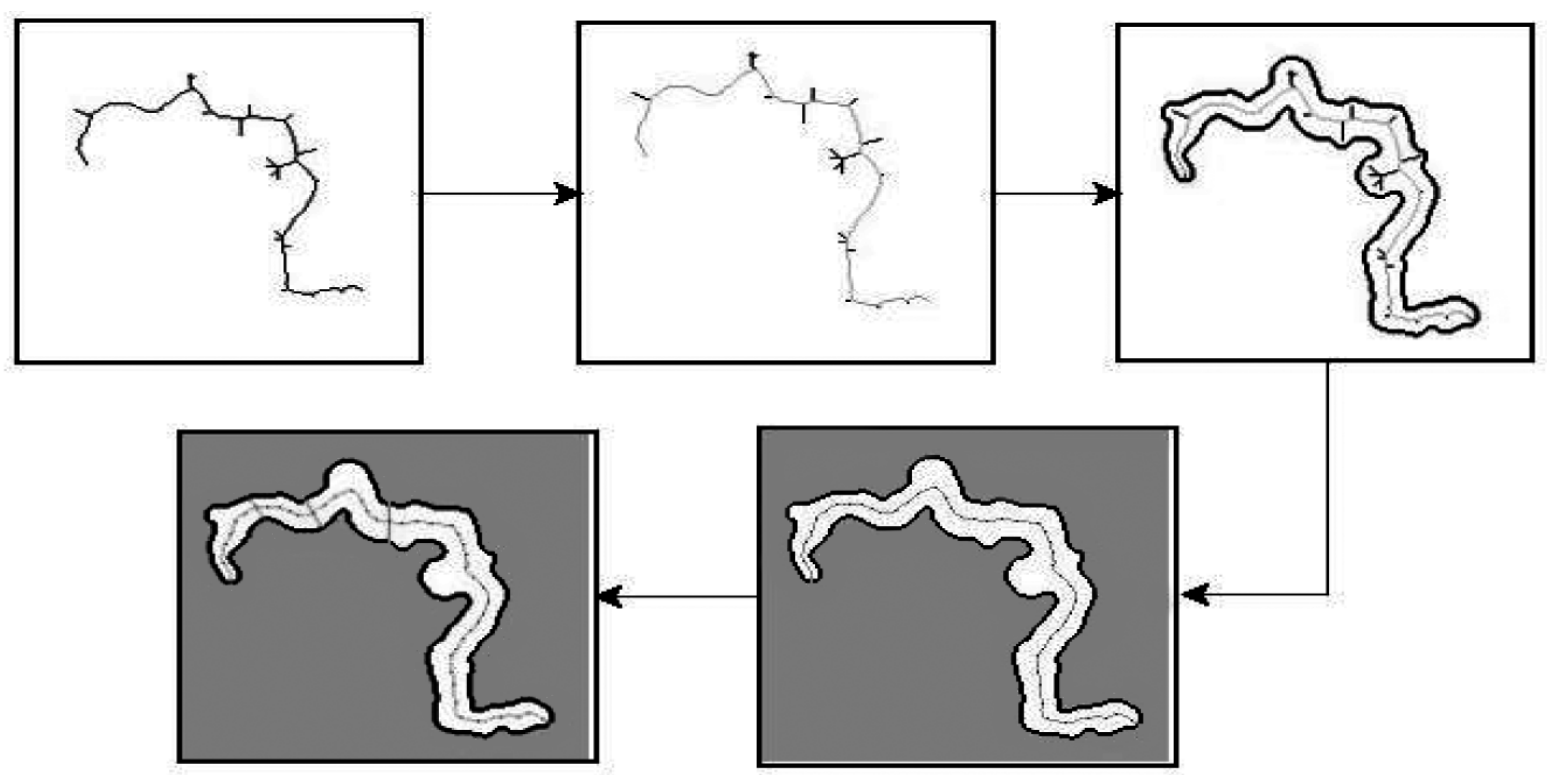

Figura 4. Diagrama de extracción de características de los objetos analizados

\section{RECONOCIMIENTO DE PATRONES PARA IDENTIFICAR "CORDONES" DE TUBERCULOSIS}

El proceso de reconocimiento de patrones en cordones de tuberculosis se ha basado en la cuantificación de las características de objetos explicadas por los expertos, como son: largo aproximadamente regular, ancho conservado, refracción en el interior del objeto y forma sinuosa. Por este motivo, cada característica geométrica se cuantificó y analizó desde el punto de vista de coherencia biológica y estadística. Como procedimiento, se realizó un estudio univariado considerando su significancia y correlación, se seleccionaron algunas variables para generar un modelo estadístico apropiado para el diagnóstico de TB basado en la identificación de cordones.

El presente modelo de clasificación de objetos ad hoc para las fotografías del CENEX TB-Trujillo fue implementado con una base de 1268 objetos, 552 positivos y 716 negativos. El modelo para diagnosticar las fotografías fue implementado con una base de 139 fotos, 52 fotos negativas y 87 fotos positivas.

Debido a las distintas condiciones de trabajo microbiológico y de digitalización referente a los cultivos MODS en el Laboratorio CENEX-Trujillo, los objetos de las imágenes digitales mostraron diferentes rangos de valores en los parámetros previamente definidos. Por este motivo, se realizó una nueva selección de objetos basado en estas fotografías, con las cuales se desarrolló un nuevo modelo estadístico para objetos y fotos.

\section{MONITOREO DEL DESEMPEÑO DEL CENEX- TRUJILLO PARA EVALUAR LA INTERVENCIÓN}

Estrategia de evaluación. Para estimar la eficiencia del presente proyecto, en la región La Libertad, se realizó el entrenamiento al personal en dos puntos primordiales: (1) Capacitación en el uso del microscopio (enfoque de muestra en medio líquido, captura de imagen, uso de los diferentes niveles de luz, campos aceptables, entre otros. (2) Capacitación en el uso del servicio WEB para el diagnóstico remoto (ingreso al servidor, envió de imágenes, recepción de resultados).

Evaluación cuantitativa. Los indicadores cuantitativos para este proyecto fueron obtenidos del monitoreo descrito y de la base de datos de casos tuberculosis resistente del Ministerio de Salud, correspondiente a la región de salud de la Libertad : (1) La proporción de imágenes digitales que son interpretables; (2) la confiabilidad del telediagnóstico comparado con las actuales medidas de control de calidad basadas en las estrategias tradicionales y lentas; (3) el tiempo desde el inicio de un tratamiento hasta el tiempo promedio de diagnóstico de tuberculosis resistente.

A las muestras procesadas con la técnica MODS también se les realizó otras pruebas de sensibilidad, ello incluyó 
Griess, en el Laboratorio Referencial de La Libertad; Genotype y prueba de sensibilidad convencional (APP), en el Instituto Nacional de Salud según la norma técnica de la Estrategia Nacional de Prevención y Control de la tuberculosis.

\section{ANÁLISIS ESTADÍSTICO}

A partir de los objetos positivos y negativos a tuberculosis, se aleatorizaron los datos y se generaron dos bases de datos independientes (base de entrenamiento y base de validación). Con la base de entrenamiento se modeló la variable dicotómica (positivo/negativo) mediante una regresión logística múltiple que incluyó las diferentes variables predictoras. El conjunto completo de variables predictoras comprendidas por las características morfológicas y de iluminación, fue primero analizado para identificar variables sobre correlacionadas, en una matriz de correlación de Spearman, ya que muchas variables no tenían distribución normal. Aquellos grupos de variables con una correlación mayor o igual a $85 \%$ fueron colapsados en una sola variable correspondiente a aquella con la mayor fuerza de asociación en una regresión logística simple, en la que se modela la probabilidad de positividad a tuberculosis de los diferentes objetos. Iniciando con un modelo saturado, se fueron eliminando las variables menos significativas una a una. Adicionalmente, iniciando con un modelo de regresión simple, incluyendo la variable más significativa, se fueron agregando las distintas variables predictoras, las cuales se conservaban en la medida que el modelo múltiple permanecía significativo.

En cada caso, los modelos anidados progresivos eran comparados mediante la prueba de máxima verosimilitud. Se analizaron los términos de interacción y los efectos no lineales de segundo orden. El mejor modelo de regresión logística múltiple obtenido a partir de la base de entrenamiento se determinó del predictor lineal respectivo. Utilizando el predictor lineal hallado, se evaluó la base de validación. Cada objeto de la base de datos de validación, de acuerdo con los valores de sus parámetros predictores, tiene un cierto valor para el predictor lineal. Utilizando distintos puntos de corte para el predictor lineal, cada objeto se clasifica como positivo o negativo, dependiendo si el valor del predictor lineal es mayor o igual que el punto de corte, o menor que el punto de corte, respectivamente. De esta manera, se obtuvieron distintos escenarios de sensibilidad y especificidad. Se identificaron los valores de sensibilidad y especificidad que maximizaron el índice de Youden, asimismo fue calculado el área bajo la curva ROC (receiving operating curve).

Para el diagnóstico automático de cada fotografía, se analizan las probabilidades de los ocho mejores objetos positivos y el número de objetos positivos de una foto. Estos datos se analizan en una regresión logística múltiple en la cual se modela la probabilidad de una foto de ser positiva para tuberculosis. De manera similar a lo realizado en la selección del modelo de objetos, se analizan las bases de datos de fotografías (base de entrenamiento y base de validación), y se determina la sensibilidad y especificidad del predictor de fotos positivas. Las proporciones de imágenes digitales interpretables fueron estimadas con 95\% intervalos de confianza. La sensibilidad y especificidad también fueron estimadas. Todos los análisis estadísticos se realizaron con una significancia del $5 \%$, utilizando el software estadístico Stata 10.

\section{RESULTADOS}

\section{MODELO CON RESPECTO AL OBJETO}

Después de la selección del mejor modelo logístico predictivo para el diagnóstico, se procedió a analizar la significancia biológica y estadística de cada variable. La Tabla 2 muestra las características de los objetos que definieron el mejor modelo logístico múltiple seleccionado.

La tabla muestra que el modelo de objeto elaborado con base en la regresión logística múltiple, tiene una alta significancia biológica, al incluir las mismas características que son usadas por los expertos humanos para la identificación de los cordones positivos, como son la longitud aproximada, espesor uniforme, refracción y forma. Este modelo es ad hoc para las imágenes de las muestras enviadas desde el laboratorio CENEX TB-Trujillo. La sensibilidad para el reconocimiento de cordones positivos a M. Tuberculosis

Tabla 2. Modelo con respecto al objeto analizado

\begin{tabular}{lc}
\hline Características & $\begin{array}{c}\text { Odds ratio }\left(\mathbf{R}^{2} ; \boldsymbol{p} \text { valor; }\right. \\
\text { coeficiente de la regresión } \\
\text { logística múltiple) }\end{array}$ \\
\hline Longitud & $(1,02 ;<0,001 ; 0,02)$ \\
\hline $\begin{array}{l}\text { Espesor desviación estándar } \\
\text { Perímetro / longitud }\end{array}$ & $(0,57 ;<0,001 ;-0,55)$ \\
$\begin{array}{l}\text { Desviación estándar de los } \\
\text { valores de iluminación media } \\
\text { del objeto }\end{array}$ & $(0,21 ;<0,001 ;-1,54)$ \\
$\begin{array}{l}\text { Media de los valores de } \\
\text { iluminación del objeto }\end{array}$ & $(0,78 ;<0,001 ;-0,24)$ \\
$\begin{array}{l}\text { Cantidad de curvas del objeto } \\
\text { por la longitud de la mayor de }\end{array}$ & $(1,08 ;<0,001 ; 0,07)$ \\
las curvas & \\
\hline
\end{tabular}


Tabla 3. Modelo con respecto a la foto de las muestras de cultivo

\begin{tabular}{lc}
\hline \multicolumn{1}{c}{ Características } & $\begin{array}{c}\text { Odds ratio } \\
\mathbf{( R}^{2}, \boldsymbol{p} \text { valor, coeficiente } \\
\mathbf{d e ~ l a ~ r e g r e s i o ́ n ~ l o g i ́ s t i c a ~} \\
\text { múltiple })\end{array}$ \\
\hline $\begin{array}{l}\text { Número de objetos de la } \\
\text { fotografía }\end{array}$ & $(0,91 ; 0,02 ;-0.089)$ \\
$\begin{array}{l}\text { Número de objetos positi- } \\
\text { vos para el modelo de foto }\end{array}$ & $(1,47,0.04 ; 0,388)$ \\
$\begin{array}{l}\text { Promedio de las } 2 \text { mejores } \\
\text { probabilidades de todos } \\
\text { los objetos de la fotografía }\end{array}$ & $(8443569 ; 0,004 ; 15,94)$ \\
\hline
\end{tabular}

fue de $92 \%$, y la especificidad de $94,9 \%$. El área bajo la curva ROC para la clasificación de objetos tipo cordones de M. tuberculosis fue 0,97 .

\section{MODELO CON RESPECTO A LA FOTO}

Después de la selección del modelo más representativo para el diagnóstico se procedió a analizar la significancia biológica y estadística de cada variable. La Tabla 3 muestra los parámetros identificados en el mejor modelo de foto seleccionado.

Del modelo logístico elaborado se obtuvo la sensibilidad y especificidad en el grupo de fotografías de las muestras con cultivos positivos y negativos. El modelo presentó una sensibilidad de 95,4\% y una especificidad de $98,1 \%$. El área bajo la curva ROC para la clasificación de objetos fue 0,99 .

\section{DISCUSIÓN}

El objetivo del presente estudio fue implementar el uso del algoritmo de diagnóstico desarrollado para el reconocimiento de los patrones de crecimiento de $M$. tuberculosis utilizando MODS; sin embargo, en un inicio, al trabajar con el algoritmo de diagnóstico para las fotografías del microscopio Olympus utilizadas en el LID-UPCH (Lima) y aplicarlo al CENEX-Trujillo se obtuvieron resultados poco alentadores; por lo que se tuvo que crear un algoritmo ad hoc para las fotografías tomadas, lo cual demostró la necesidad de realizar ajustes en ciertos parámetros como iluminación, zoom, día de mejor crecimiento del cultivo, los cuales son diferentes para cada laboratorio, y así obtener la estandarización de la prueba.

Para solucionar estas limitaciones se analizaron los resultados iniciales de los objetos mal clasificados por el software, se asumió que dicho problema se debió a diversos motivos: 1) Las fotografías enviadas no estaban dentro de los parámetros establecidos; 2) Las muestras no correspondían a la presencia de cordones; 3) Los objetos seleccionados del CENEX-Trujillo tenían una variación de luminosidad muy alta comparada con las fotos que sirvieron para el modelamiento del software En el LID-UPCH; 4) La implementación en el algoritmo inicial de procesamientos iterativos para disminución del contraste en diferentes rangos mejoraron la sensibilidad, pero fueron aún muy sensibles a los cambios de fuente de luz, por lo que se procedió a las siguientes soluciones: a) Se realizó el cultivo de nuevas muestras mediante el proceso MODS en el laboratorio del CENEX-Trujillo; b) Se adquirieron las imágenes en el periodo adecuado de crecimiento, es decir, 10 días bajo las mismas condiciones de zoom e iluminación; c) Se programó nuevas características de iluminación para el procesamiento de las fotografías y así compensar la variación de la fuente de luz en Trujillo (CENEXTrujillo) y Lima (LID-UPCH). Finalmente, se reevaluaron los datos después de estos cambios, de manera $a d$ hoc para las fotografías del CENEX TB de Trujillo, de donde se obtuvo resultados con una sensibilidad de 92,0 , y una especificidad de 94,9 para el nivel objeto; la sensibilidad fue de 95,4 y la especificidad de 98,1 con respecto a la foto. Esta estandarización del algoritmo de reconocimiento ad hoc permitió hacer el diagnóstico de tuberculosis a partir de imágenes digitales provenientes de un cultivo MODS del CENEX-Trujillo.

En el presente estudio, el valor obtenido para la curva ROC, con respecto al objeto, fue de 0,97 ; mientras que en el área bajo la curva a con respecto a la foto fue de 0,99 , lo que demuestra que el modelo usado para el diagnóstico fue adecuado, por encontrarse dicho valor cercano a $1^{(20)}$. En el modelo de predicción propuesto, el índice de Youden fue de 0,86 con respecto al objeto, y de 0,93 con respecto a la foto, lo cual muestra que cumple con este parámetro para poder evaluar la bondad del modelo logístico. Una medida conjunta de eficiencia de un medio diagnóstico fue propuesta por W.J. Youden en 1950, el índice Youden (IJ), el cual refleja la diferencia entre la tasa de verdaderos positivos y la de falsos positivos. Un buen test debe tener alta esta diferencia. Teóricamente, es igual a 1 solo cuando la prueba diagnóstica es perfecta, es decir, cuando $S+E=2$, de modo que también puede decirse que cuánto más cercano a 1 , mejor es la prueba diagnóstica que se está evaluando. Este índice tiene la ventaja de no estar afectado por la selección de la prevalencia, y es preferido por la combinación de los sencillos valores de sensibilidad y especificidad. Se considera que una prueba diagnóstica no es adecuada si su índice de Yoden es menor o igual a cero ${ }^{(21)}$.

Para discriminar correctamente de una forma más coherente los objetos, uno se debe basar en 
observaciones de expertos en lecturas de la prueba de sensibilidad MODS, tal como se hizo en este estudio al considerar el apoyo de la especialista Luz Caviedes quien distinguía fotografías de cultivos positivos y de cultivos negativos. Cabe resaltar que el descubrimiento de las características de crecimiento de detección del M. tuberculosis, que llevó al desarrollo de MODS, se atribuye a la bióloga Luz Caviedes, que trabajó bajo la supervisión del Dr. Robert Gilman en el Laboratorio de Tuberculosis de la Facultad de Ciencias de la Universidad Peruana Cayetano Heredia, Lima, Perú (22).

En el presente trabajo, el método MODS demostró ser una buena prueba para diagnosticar TB y TBMDR con gran sensibilidad y especificidad al décimo día. El conocimiento oportuno del patrón de resistencia es imprescindible; luego de iniciado el esquema primario de tratamiento, este debe ser corroborado con la prueba de sensibilidad para confirmar si es adecuado o debe modificarse según el patrón de resistencia para así conseguir la curación del paciente ${ }^{(23)}$. En este estudio referente al telediagnóstico se logró una sensibilidad de 92,0\% y una especificidad de $94,9 \%$ con respecto al objeto, y una sensibilidad de $95,4 \%$ y una especificidad de $98,1 \%$ con respecto a la foto. En un estudio anterior se demostró que la transmisión de imágenes digitales MODS utilizando la telefonía móvil dio lugar a una forma sencilla de diagnosticar la tuberculosis remotamente por un técnico humano ${ }^{(24)}$.
El algoritmo de reconocimiento de patrones MODS obtenido en el presente estudio, que mostró alta sensibilidad y especificidad, podría ser utilizado en un mayor alcance para diagnosticar la tuberculosis de forma remota y para detectar multidrogorresistencia a partir de imágenes digitales transmitidas. Si bien es cierto que en los laboratorios microbiológicos de TB donde se realiza MODS existen técnicos calificados en la lectura MODS, es importante resaltar que en el uso del TB-MODS por técnicos con menos experiencia, la evaluación de muestras problemáticas pueden generarles dudas, en cuyo caso, una ayuda a distancia para interpretar un cultivo MODS también es útil. En conclusión, el presente trabajo demuestra que es posible el uso del telediagnóstico de tuberculosis en lugares remotos, lo cual podría contribuir con el diagnóstico temprano de TB y TB-MDR.

Agradecimientos: al personal del laboratorio del CENEX-Trujillo por su colaboración en la realización de la prueba de sensibilidad MODS que sirvieron para la ejecución del presente estudio.

In memóriam: Luz Caviedes fue científica peruana que descubrió el MODS y por primera vez reconoció el potencial de emplear su observación del conocimiento rápido de las características de órdenes de $M$. tuberculosis en un cultivo líquido en una prueba de diagnóstico. Lucy falleció el 4 de noviembre del 2012.

Conflictos de interés: los autores declaran no tener conflictos de interés.

Fuentes de financiamiento: el presente estudio fue financiado por el proyecto CONCYTEC-PROCYT 324-2011.

\section{REFERENCIAS BIBLIOGRÁFICAS}

1. Bonilla C. Situación de la tuberculosis en el Perú. Acta Med Per. 2008 JulSet;25(3):163-70.

2. Robledo J, Mejía G. Actualidad en el diagnóstico de tuberculosis por el laboratorio. Infectio 2001;5(4):251-9.

3. Park WG, Bishai WR, Chaisson ER, Dorman SE. Performance of the microscopic observation drug susceptibility assay in drug susceptibility testing for Mycobacterium tuberculosis. J Clin Microbiol. 2002 Dec;40(12):4750-2.

4. Moore DA, Mendoza D, Gilman RH, Evans CA, Holim Delgao MG, Guerra J. et al. Microscopic observation drug susceptibility assay, a rapid, reliable diagnostic test for multidrug-resistant tuberculosis suitable for use in resourcepoor settings. J Clin Microbiol. 2004 Oct; $42(10): 4432-7$.

5. Moore DA, Caviedes L, Gilman RH, Coronel J, Arenas F, LaChira D, et al. Infrequent MODS TB culture cross-contamination in a high-burden resource-poor setting. Diagn Microbiol Infect Dis. 2006 Sep;56(1):35-43.

6. Moore DA, Evans CA, Gilman RH, Caviedes L, Coronel J, Vivar $\mathrm{A}$, et al. Microscopic-observation drug-susceptibility assay for the diagnosis of TB. N Engl J Med 2006 Oct;355(15):1539-50.

7. Oberhelman RA, Soto-Castellares G, Caviedes L, Castillo ME, Kissinger P, Moore DA, et al. Improved recovery of Mycobacterium tuberculosis from children using the microscopic observation drug susceptibility method. Pediatrics. 2006 Jul;118(1):e100-6.

8. Caviedes L, Lee TS, Gilman RH, Sheen P, Spellman E, Lee EH, et al. Rapid, efficient detection and drug susceptibility testing of Mycobacterium tuberculosis in sputum by microscopic observation of broth cultures. The Tuberculosis Working Group in Peru. J Clin Microbiol. 2000 Mar;38(3):1203-8.

9. Vargas D, García L, Gilman RH, Evans C, Ticona E, Navincopa M , et al. Diagnosis of sputum-scarce HIVassociated pulmonary tuberculosis in Lima, Peru. Lancet. 2005 Jan 8-14;365(9454):150-2.

10. Arias M, Mello FC, Pavón A, Marsico AG, Alvarado-Gálvez C, Rosales S, et al. Clinical evaluation of the microscopicobservation drug-susceptibility assay for detection of tuberculosis. Clin Infect Dis. 2007 Mar 1;44(5):674-80.

11. Ha DT, Lan NT, Kiet VS, Wolbers M, Hang HT, Day J, et al. Diagnosis of pulmonary tuberculosis in HIVpositive patients by microscopic observation drug susceptibility assay. J Clin Microbiol. 2010 Dec;48(12):4573-9. doi: 10.1128/ JCM.00687-10. 
12. Ha DT, Lan NT, Wolbers M, Duong TN, Quang ND, Thi Van Thinh T, et al. Microscopic observation drug susceptibility assay (MODS) for early diagnosis of tuberculosis in children. PLoS One. 2009 Dec 17;4(12):e8341. doi: 10.1371/journal.pone.0008341.

13. Shiferaw G, Woldeamanuel Y, Gebeyehu M, Girmachew F, Demessie D, Lemma E. Evaluation of microscopic observation drug susceptibility assay for detection of multidrug-resistant Mycobacterium tuberculosis. J Clin Microbiol. 2007 Apr;45(4):1093-7.

14. Kaufman DR, Patel VL, Hilliman C, Morin PC, Pevzner J, Weinstock RS, et al. Usability in the real world: assessing medical information technologies in patients' homes. J Biomed Inform. 2003 Feb-Apr;36(1-2):45-60.

15. Handels H, Rinast E, Busch C, Hahn C, Kühn V, Miehe J, et al. Image transfer and computer-supported cooperative diagnosis. J Telemed Telecare. 1997;3(2):103-7.

16. Alva A, Aquino F, Gilman RH, Olivares C, Requena D, Gutiérrez AH, et al. Morphological characterization of Mycobacterium tuberculosis in a MODS culture for an automatic diagnostics through pattern recognition. PLoS One. 2013 Dec 16;8(12):e82809. doi: 10.1371/ journal.pone.0082809.

17. Blaya JA, Fraser HS, Holt B. E-health technologies show promise in developing countries. Health Aff (Millwood). 2010 Feb;29(2):244-51. doi: $10.1377 /$ hlthaff.2009.0894.

18. Gonzalez RC, Woods RE. Digital Image Processing. Reading, MA: Addison-Wesley; 1992.

19. Parker JR. Algorithms for image processing and computer vision. Indianapolis: Wiley Publishing, INC.; 1997.

20. Cerda J, Cifuentes L. Uso de curvas ROC en investigación clínica: Aspectos teórico-prácticos. Rev Chil Infect. 2012 Abr;29(2):138-41.

21. Zhou H. Statistical Inferences for the Youden Index. Tesis para obtener el grado de Doctor en Filosofía. Georgia State University. Atlanta, Estados Unidos. 2011.
22. Caviedes L, Moore DA. Introducing MODS: a low-cost, low-tech tool for high-performance detection of tuberculosis and multidrug resistant tuberculosis. Indian J Med Microbiol. 2007 Apr;25(2):87-8.

23. Mendoza-Ticona A, Moore DAJ, Alarcón V, Samalvides F, Seas C. Propuesta de esquemas de tratamiento antituberculosis basados en la susceptibilidad a isoniacida y rifampicina. Rev Peru Med Exp Salud Publica. 2013;30(2):197-204.

24. Zimic M, Coronel J, Gilman RH, Luna CG, Curioso WH, Moore DA. Can the power of mobile phones be used to improve tuberculosis diagnosis in developing countries?. Trans R Soc Trop Med Hyg. 2009 Jun;103(6):63840. doi: 10.1016/j.trstmh.2008.10.015.

Correspondencia: Mirko Zimic Peralta. Dirección: Calle Bosovich 323, Dpto. 201

Teléfono: (+511) 224-5118

Correo electrónico:mirko.zimic@upch.pe

\section{Consulte las ediciones anteriores de la} Revista Peruana de Medicina Experimental y Salud Pública en

\section{wwW.scielosp.org}

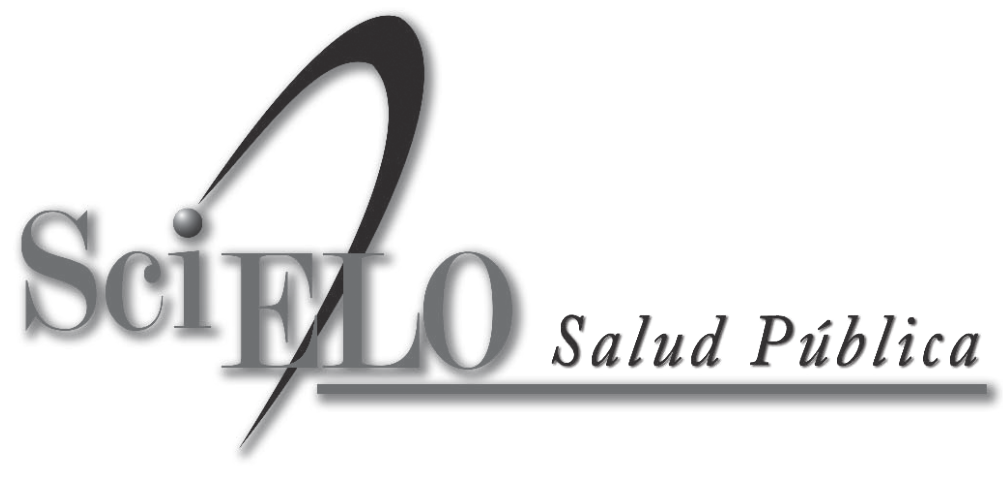

